\title{
Primary meningioma of the middle ear: case report
}

\author{
M George, C IKonomidis, M Pusztaszeri, P Monnier
}

\begin{abstract}
Objective: To report a rare case of primary meningioma of the middle ear.

Method: We describe the case of a 55-year-old woman who presented with decreased hearing and fullness in the left ear, with a middle-ear mass. We also review the world literature regarding primary extracranial meningioma of the middle ear and its management.

Results: Primary middle-ear meningioma, a rare clinical entity, was diagnosed in this patient based on an initial transmastoid biopsy. Magnetic resonance imaging with gadolinium enhancement excluded the presence of an intracranial component. Complete excision of the tumour was achieved using a combined approach tympanoplasty. The patient had an uneventful post-operative course.

Conclusion: Meningiomas, although rare in the middle ear, need to be included in the differential diagnosis of middle-ear lesions presenting to the otolaryngologist. This case emphasises the management strategy required when dealing with a middle-ear mass.
\end{abstract}

Key words: Middle Ear; Meningioma; Mastoid

\section{Introduction}

Meningioma make up $18 \%$ of primary intracranical tumors though it is rarely identified as a primary middle-ear lesion without an intracranial component. The estimated incidence of meningioma occurring extracranially is 2 per cent, and its occurrence in the middle ear is extremely rare. ${ }^{1}$ Intracranial meningiomas can extend to the middle-ear cleft via the tegmen tympani, posterior fossa plate, internal auditory canal or jugular foramen. Most reported cases initially regarded as primary middle-ear meningiomas were eventually shown to have an intracranial origin. ${ }^{2-4}$ Most of these cases were reported before magnetic resonance imaging (MRI) with gadolinium enhancement became widely available.

This case report emphasises several important factors in the diagnosis and treatment of primary extracranial meningioma. The accompanying literature review also highlights salient features of this tumour.

\section{Case report}

A 55-year-old woman presented with a three-year history of decreased hearing and a sensation of fullness in the left ear. The symptoms were aggravated after alcohol intake or increased physical activity.

On otoscopy, the external auditory canal was found to be normal, but visualisation of the posterosuperior quadrant of the middle ear showed a mass behind an intact tympanic membrane (Figure 1). Audiometry showed a moderate conductive hearing loss. High resolution computed tomography (CT) scanning (Figure 2) and subsequent MRI with gadolinium enhancement (Figure 3) revealed a lesion in the epitympanum and mastoid antrum. The mass was confined to the mastoid antrum and epitympanum, with an intact ossicular chain, facial canal and otic capsule. The radiological appearances were nonspecific, and a tentative diagnosis of schwannoma was made.

A transmastoid biopsy of the lesion was performed using a postaural approach. Histological examination of the biopsy specimen was consistent with meningioma.

After the biopsy, the patient's general condition was normal, and neurological examination showed no abnormality. Re-evaluation of the MRI confirmed no intracranial extension of the tumour.

A combined approach tympanoplasty was performed. The tumour was removed in toto. The lesion was nonvascular and filled the attic and antrum (Figure 4). There was no evidence of infiltration of the ossicles or tympanic membrane by the tumour. The sinus plate, lateral semicircular canal and tegmen were intact. The head of the malleus and incus were removed to facilitate complete elimination of the tumour. Hearing reconstruction was achieved by refashioning the head of the malleus and replacing it over the stapes head.

The patient did well after surgery, with no evidence of tumour recurrence at three months' follow up.

\section{Histopathology}

Macroscopic examination showed an encapsulated, $2 \times 1 \times$ $0.5 \mathrm{~cm}$ lesion. Histological examination showed a proliferation of bland, meningothelial-like cells forming diffuse sheets admixed with fibrous tissue. Nuclei were found with occasional pseudoinclusions and small nucleoli. No mitotic activity or necrosis was seen. Evidence of microcalcification was present. Periodic acid Schiff staining was negative.

Immunohistochemical analysis showed positive staining for vimentin, pancytokeratins (AE1AE3) and epithelial 


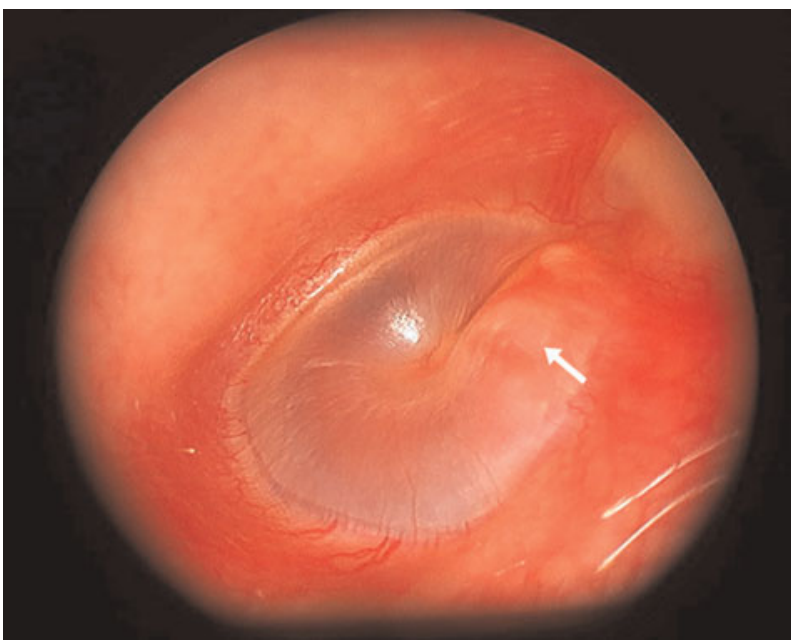

FIG. 1

Otoscopic photograph showing a fullness (arrow) in the posterosuperior quadrant of the tympanic membrane.

membrane antigen, but immunonegativity for S-100, smooth muscle actin and progesterone receptors. The MIB-1L1 labelling index was very low (less than 2 per cent).

The morphological and immunohistochemical characteristics of the tumour were consistent with a meningioma (World Health Organization (WHO) grade one) (Figure 5).

\section{Discussion}

The occurrence of meningioma in the middle ear as a primary lesion is very rare, with few reported cases. Nager classified temporal bone meningiomas into two main types: type one (the commoner), representing extension of an intracranial meningioma; and type two, occurring without intracranial extension. ${ }^{5}$ The rare type two entity should be diagnosed only after confirmatory MRI. Most of the published case reports are from the pre-MRI era, with insufficient evidence to exclude the possibility of extension from a primary intracranial tumour. There are very few reported cases of primary middle-ear

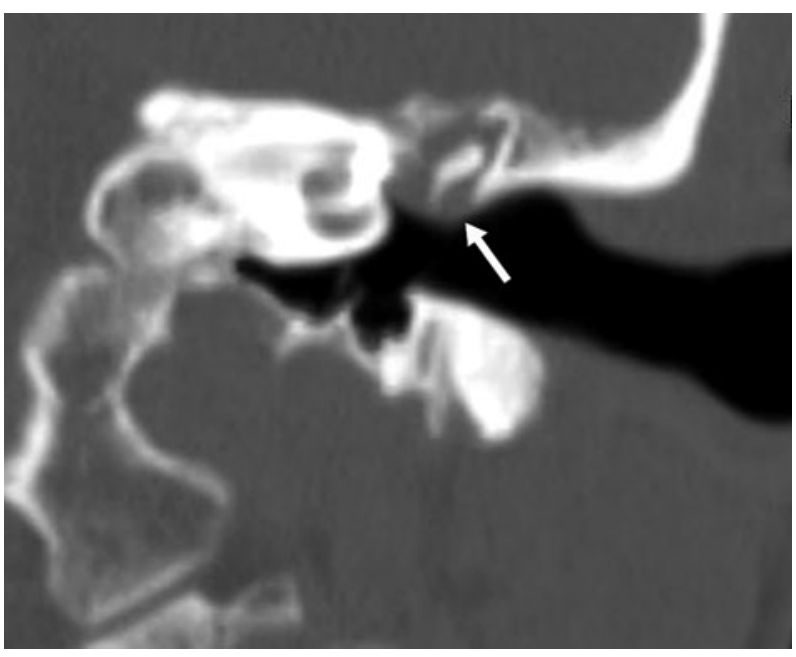

FIG. 2

Coronal, high resolution computed tomography scan of the left temporal bone, demonstrating a soft tissue mass (arrow) in the middle ear, with no evidence of bony erosion.

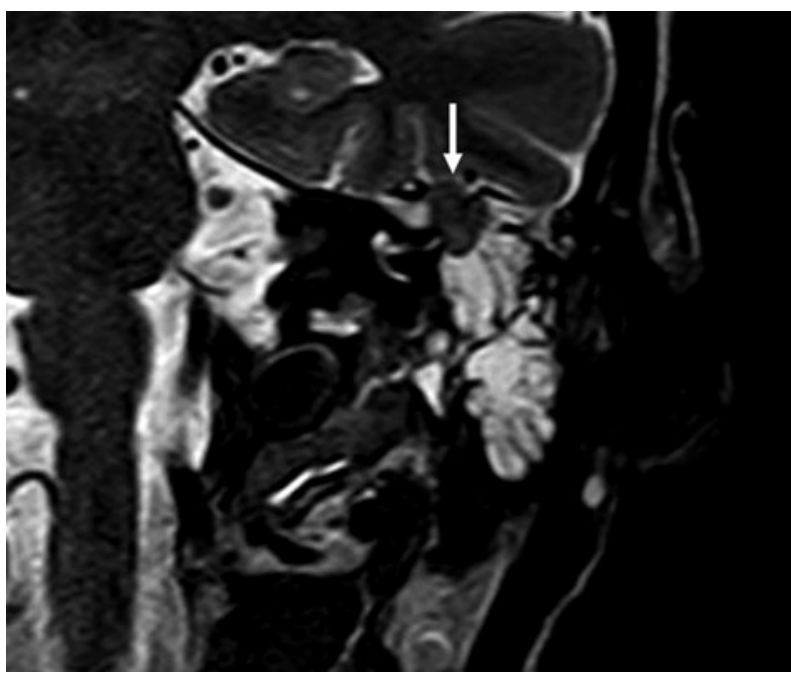

FIG. 3

Coronal, T2-weighted magnetic resonance imaging scan with gadolinium enhancement, showing an isointense, extra-axial mass (arrow) within the petrous bone, inferior to the temporal basal dura. The lesion is in the superior aspect of the fluid-filled mastoid air cells, just lateral to the semicircular canal.

meningioma without intracranial extension. ${ }^{6,7}$ Thompson et al. described 36 cases of temporal bone meningioma, but were not able to exclude intracranial extension due to the absence of proper radiological evaluation. ${ }^{8}$ An MRI with gadolinium enhancement is superior to plain X-ray or CT scan for differentiating peripheral extension of an intracranial meningioma from the less common primary extracranial meningioma (as encountered in our patient). ${ }^{3,8}$

The differential diagnosis of a middle-ear mass includes schwannoma, glomus tumour, metastatic tumour and ceruminous gland tumour. Differentiation of middle-ear meningioma from facial nerve schwannoma is clinically difficult, due to these tumours' similar clinical presentations. Both tumours predominantly affect women aged 45-55 years, and present with symptoms of hearing loss and tinnitus. In addition, there are no characteristic radiological differences between the two tumours. ${ }^{9}$ Therefore, the

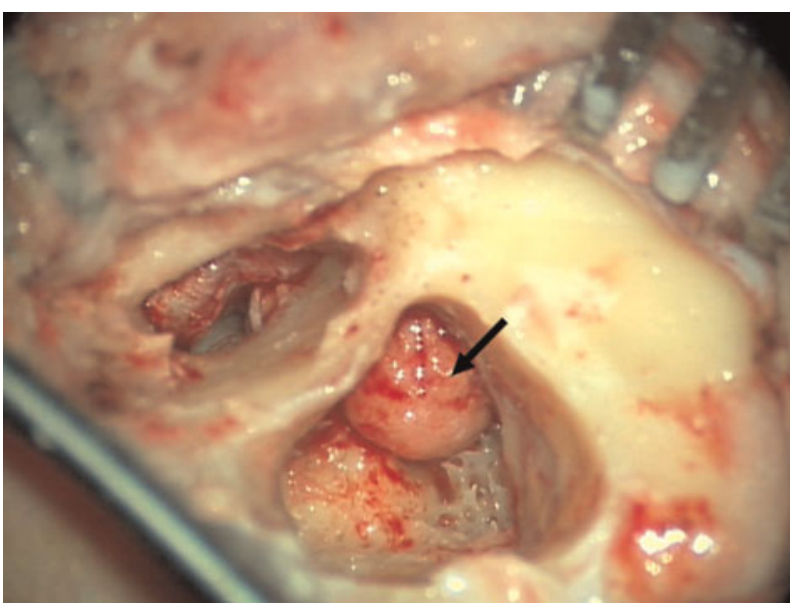

FIG. 4

Intra-operative photograph showing the meningioma in the epitympanum, aditus and antrum (arrow). No erosion of the tegmen or sinus plate was seen. 


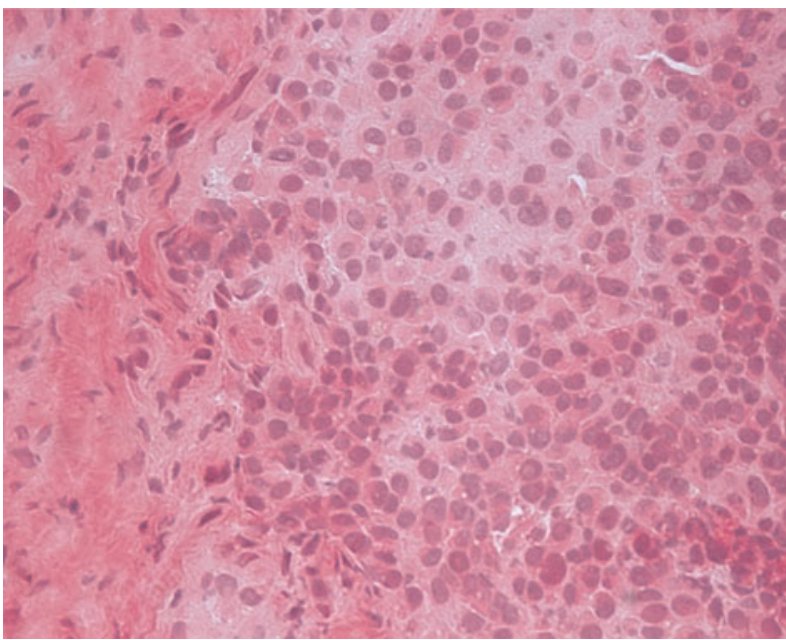

FIG. 5

Photomicrograph of the meningioma showing proliferation of bland, meningothelial-like cells forming diffuse sheets admixed with fibrous tissue. Nuclei were found with occasional pseudoinclusions and small nucleoli. No mitotic activity or necrosis was seen $(\mathrm{H} \& \mathrm{E} ; \times 40)$.

diagnosis can only be confirmed after histopathological examination, as the clinical symptoms and radiological signs are usually nonspecific, as in our patient.

Meningiomas are usually benign in nature, however, malignant transformation is possible in some patients. Histopathological examination of our patient's specimen revealed a mixed, grade one, benign meningioma (based on WHO classification). Other types include atypical (grade two) and anaplastic (grade three). The meningotheliomatous or transitional variant of grade one is more often associated with extracranial meningioma. ${ }^{4}$ The immunohistochemical profile of meningioma is quite characteristic, and is useful in differentiating these tumours from schwannoma. It has been noted that immunoreactivity for epithelial membrane antigen and keratin will be strongly positive in meningiomas, but negative or only weakly positive in schwannomas. ${ }^{10}$

- Meningiomas make up 18 per cent of primary intracranial tumours, whereas the estimated incidence of extracranial meningiomas is only 2 per cent. Meningioma in the middle ear without intracranial extension is extremely rare

- In cases of primary middle-ear meningioma, total excision of the lesion remains the mainstay of management

- Meningioma needs to be included in the differential diagnosis of middle-ear lesions

Complete surgical excision is the mainstay of management for primary middle-ear meningiomas. Surgery is difficult in the majority of cases due to the infiltrative nature of the tumour along the Haversian canals, and the absence of clear margins. However, complete excision of the lesion was possible in our patient, although removal of the head of the malleus and incus was temporarily required to facilitate tumour excision. We did not notice any erosion of the ossicles or tegmen plate.

As meningiomas are slow-growing tumours, long term follow up with MRI scanning is important to exclude recurrence. A change from conductive to sensorineural hearing loss may be the earliest sign of progressive disease; such a change may indicate erosion of the bony labyrinth, which is often spared in the early part of the disease. ${ }^{11}$ In our patient, follow-up pure tone audiometry revealed improved hearing. The role of radiation therapy for residual tumours is unclear, due to the rare occurrence of these tumours. ${ }^{12}$

\section{Conclusion}

Primary middle-ear meningioma is a rare clinical entity, with nonspecific clinical and radiological features. Histopathological and immunohistochemical findings are helpful in differentiating it from other, more common middle-ear tumours, such as schwannoma and glomus tympanicum. An increased awareness among otolaryngologists is necessary in order to correctly diagnose this rare but well reported tumour. Long term follow up using MRI with gadolinium enhancement is necessary to exclude recurrence, which can occur even after complete surgical excision.

\section{References}

1 Friedman CD, Costantino PD, Teitelbaum B, Berktold RE, Sisson GA Sr. Primary extracranial meningiomas of the head and neck. Laryngoscope 1990;100:41-8

2 Maniglia AJ. Intra and extracranial meningioma involving the temporal bone. Laryngoscope 1978;88:1-58

3 Parisier SC, Som PM, Shugar JM, Marovitz WF. The evaluation of middle ear meningiomas using computerized tomography. Laryngoscope 1978;88:1170-7

4 D'Angelo AJ, Marlowe A, Marlowe FI, Mobini J. Primary meningioma of the middle ear: do they exist? Ear Nose Throat J 1991;70:794-8

5 Nager GT. Meningiomas involving the temporal bone: clinical and pathological aspects. Ir J Med Sci 1966;6:69-96

6 Uppal HS, Kabbani M, Reddy V, Kaur S. Ectopic extracranial meningioma presenting as an aural polyp. Eur Arch Otorhinolaryngol 2003;260:322-4

7 Kumar G, Basu S, Sen P, Kamal SA. Ectopic meningioma: a case report with a literature review. Eur Arch Otorhinolaryngol 2006;263:426-9

8 Thompson LD, Bouffard IP, Sandbeg GD, Mena H. Primary ear and temporal bone meningiomas: a clinicopathologic study of 36 cases with a review of the literature. Mod Pathol 2003;31:510-13

9 Hilton MP, Kaplan DM, Ang L, Chen JM. Facial nerve paralysis and meningioma of the internal auditory canal. J Laryngol Otol 2002;116:132-4

10 Borin A, Okanda MD, Cruz OLM. Intracanalicular meningioma diagnostic by immunohistochemistry. Rev Bras Otorrinolaryngol 2008;74:320

11 O'Reilly RC, Kapadia SB, Kamerer DB. Primary extracranial meningioma of the temporal bone. Otolaryngol Head Neck Surg 1998;118:690-3

12 Ferlito A, Devaney KO, Rinaldo A. Primary extracranial meningioma in the vicinity of the temporal bone: a benign lesion which is rarely recognized clinically. Acta Otolaryngol 2004;124:5-7

Address for correspondence:

Dr Mercy George,

Department of Otolaryngology Head and Neck Surgery,

Centre Hospitalier Universitaire Vaudois,

Lausanne 1011, Switzerland.

Fax: +41213142607

E-mail: roymercy@hotmail.com

Dr M George takes responsibility for the integrity of the content of the paper.

Competing interests: None declared 